\title{
NOTE FOR THE READER
}

he book you are about to read requires some preliminary clarifications.

1 First, this essay proposes an agreement with the reader, a request for engagement. I ask for a willingness to be open and exposed to different movements of language as an ongoing experience of reading. I have conceived this text as a sort of wayfaring, and I suggest approaching the experience of drinking a glass of wine in the same way: each path followed, each sip ingested, can offer different thoughts and different feelings. Like a glass of wine, moreover, the text can be understood and felt in different ways, depending on the background of the reader. Inviting openness to multiple perspectives is the main reason why names and notes are reduced to a minimum.

Second, the essay comprises two parts, representing two different periods of the journey. These periods differ in time: the first was written between 2015 and 20I6, the second in 2017 and 2018. In the Italian version, they correspond to two different books, published, respectively, in 2016 and 20I8. This temporal gap entails not only the appearance, in the second part, of new issues as well as the deepening of some already presented in the first but also a clear difference in the form and style of writing. 
The first part is more belligerent and dry, the second calmer and smoother. But they both advance the same arguments, and I find the sequence a good way to communicate to the reader how this experience of thinking and feeling with wine came into being.

Third, regarding the title: epistenology is my neologism, and it indicates two different semantic domains. One clearly relates to the crasis between epistemology and oenology: the theory of knowledge and the knowledge of wine condensed into one word. The other semantic domain, however, also plays a pivotal role in the book: the complex combination of epistemology with ontology, where the $n$ of ontology has replaced the $m$ of epistemology. In my view, this expresses the idea that knowledge and being are never fully separable; rather, they relate to each other. So the title expresses the following: through wine, we try to show how knowledge and appreciation are always participative and intimate actions that make the reality as we perceive it. As with life, wine is what we constantly make of it. This is not a subjective position but rather a radically relational one: I try to approach wine as an encounter, a continuous correspondence of doing and undergoing. Epistenology proposes precisely an ecology of attention-as caring, listening, and making-with wine. 